\title{
A low-cost experimental rig for multi-DOF unsteady thrust measurements of aquatic bioinspired soft robots
}

\author{
Artur K. Lidtke ${ }^{1}$, Francesco Giorgio-Serchi ${ }^{1}$, Matt Lisle ${ }^{1}$ and Gabriel D. Weymouth ${ }^{1}$
}

\begin{abstract}
The design, calibration and testing of an experimental rig for measuring 2-DOFs unsteady loads over aquatic robots is discussed. The presented apparatus is specifically devised for thrust characterization of a squid-inspired soft unmanned underwater vehicle, but its modular design lends itself to more general bioinspired propulsion systems and the inclusion of additional degrees of freedom. A purposely designed protocol is introduced for combining calibration and error compensation upon which force and moment measurements can be performed with a mean error of $0.8 \%$ in steady linear loading and $1.7 \%$ in unsteady linear loading, and mean errors of $10.2 \%$ and $9.4 \%$ respectively for the case of steady and dynamic moments at a sampling rate of the order of $10 \mathrm{~Hz}$. The ease of operation, the very limited cost of manufacturing and the degree of accuracy make this an invaluable tool for fast prototyping and low-budget projects broadly applicable in the soft robotics community.
\end{abstract}

\section{INTRODUCTION}

Soft robots are becoming ubiquitous [1], but the aquatic environment is one context where they could thrive. Indeed, the lack of rigid supportive structures represents less of a hindrance due to the equivalence between the density of the ambient fluid and that of the elastomeric materials of which these robots are typically composed. Evidence of this is found in nature, where a large number of aquatic organisms exist that are partly or entirely devoid of skeletal structures. The growing interest from the robotics community for fish [2], [3], medeusae [4] and molluscs [5], [6] and other softbodied sea-dwelling organisms suggests that a proliferation of new aquatic bioinspired soft robots is likely to occur.

However, while the dense medium partly makes up for the lack of rigid structures, the utilization of nature-inspired complex morphologies [7], [8], [9] and modes of propulsion requires that adequate diagnostic facilities are developed in order to perform comprehensive kinematics and dynamics analysis of new prototypes [10], [11], [12]. On the one hand, the growing interest for multi-limbed and modular systems prescribes the need for dedicated sensor platforms capable of simultaneously measuring the loads exerted over several degrees-of-freedom (DOFs). On the other hand, the locomotion routines of these biomimetic platforms have, in most cases, a prominent time-varying component [13], thus making it necessary to account for the temporal dimension in

*This work was supported by the Lloyd's Register Foundation, the Natural Environment Research Council (grant NE/P003966/1), the Southampton Marine and Maritime Institute under the SMMI-HEIF grant and the NEXUSS-CDT

${ }^{1}$ A. K. Lidtke, F. Giorgio-Serchi, M. Lisle and G.D. Weymouth are with the Fluid-Structure-Interaction Research Group, Faculty of Engineering and the Environment, University of Southampton, Southampton, UK f.giorgio-serchidsoton.ac.uk the analysis of their signal. Finally, one asset of soft robots is the ability to design low-cost, often disposable prototypes, as well as their suitability for student projects. This suggests that a diagnostic facility of this kind should ideally fall within a cost scale comparable to that of the robots themselves.

With this focus in mind, we have designed a low-cost experimental apparatus for measuring the thrust produced by a bioinspired aquatic soft robot and tested it with a prototype of such vehicle. This facility enables the measurement of loads exerted in 2-DOFs, namely thrust along the surge axis and moment around the yaw axis of the robot. Because timedependent loads are deemed particularly important in the propulsion routine entailed with these kind of robots, we dedicate particular care to the calibration of this apparatus in dynamic conditions, thus ensuring an all-time constrain of the associated errors.

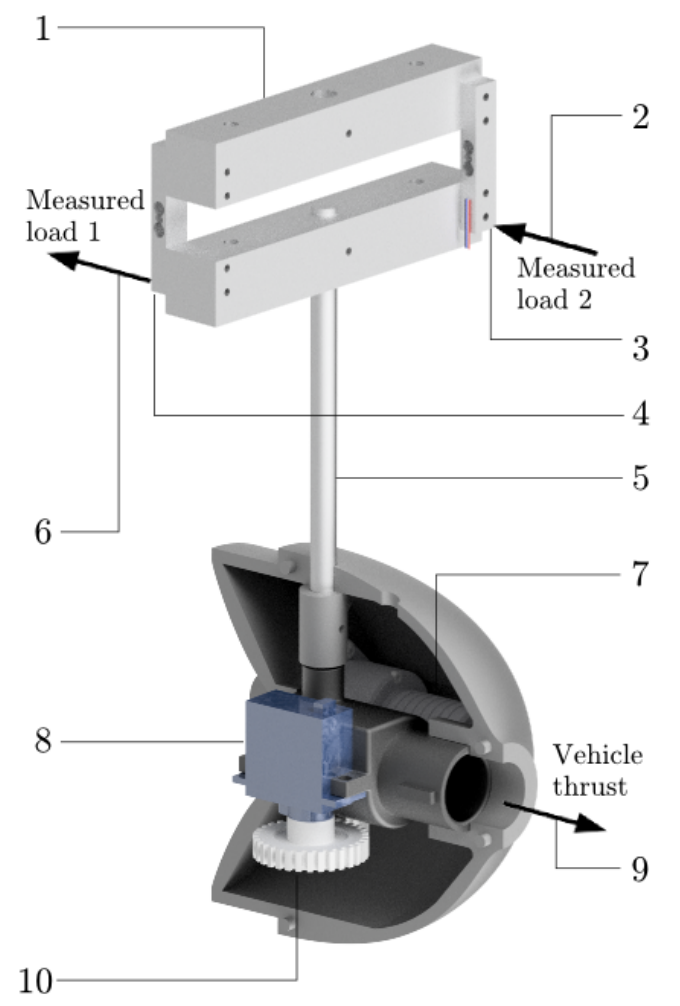

Fig. 1. Schematic of the experimental rig and the attachment point of the robot. Items refer to: (1) support blocks for the transducers, (2) direction of load on transducer 1, (3) transducer 1, (4) transducer 2, (5) rod for prototype support, (6) direction of load on transducer 2, (7) centrifugal pump, (8) servo motor, (9) direction of flow issuing from the nozzle, (10) spur gear for valve actuation. 


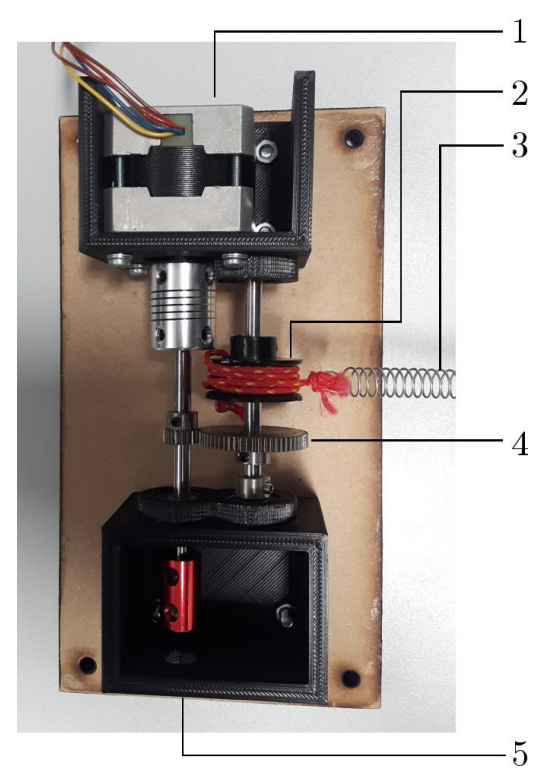

Fig. 2. Top view of the apparatus for the calibration: (1) stepper motor, (2) pulley, (3) spring, (4) spur gear for motor-pulley coupling, (5) rotary encoder.

\section{MEASUREMENT RIG DESIGN}

The apparatus consists of a platform suspended over the experimental water tank, Fig. 1, held in place by aluminium bars, item (3) in Fig. 3 (a). A vertically oriented rigid cylindrical rod $8.0 \mathrm{~mm}$ in diameter, item (5) in Fig. 1, links the submerged robotic prototype to the measuring unit. The entire apparatus fits in a $150 \times 300 \times 300 \mathrm{~mm}$ volume, making it easily transportable. The measuring unit is composed of two strain gauge-instrumented CZL616C load cells, rated for maximum load of $780 \mathrm{~g}$ each, mounted in a perpendicular orientation to the horizontal plane of the robot. The twin transducers are fitted at a known relative distance, see (3) and (4) in Fig. 1, and are supported by central aluminium block marked as (1) in Fig. 1. From the shear exerted over each transducer, (2) and (6) in Fig. 1, the load in the direction of the shear can be derived; while difference in the observed shear between two homologous transducers is interpreted as a moment around the axis normal to the exerted load. Each couple of transducers thus enables the acquisition of loads in 2-DOF. By adding two additional couples of transducers a 6-DOF system can readily be achieved and thanks to its lightweight and compact nature, the system can be conveniently mounted on to a moving platform for drag measurement and self-propulsion tests.

Each of the load cells is excited with a $5 \mathrm{~V}$ voltage, which is transformed into a voltage drop linearly proportional to the applied load via the use of a Wheatstone bridge of strain gauges. The output signal needs to be amplified in order to be recorded, which is currently done using HX711 24-Bit Analog-to-Digital Converters (ADC). These are off-the-shelf integrated circuits intended for use in precision weight scales but have been successfully applied in the present context. Their advantage is the high precision, ease of integration and low cost. They do, however, limit the acquisition rate due to the circuit relying on the on-chip oscillator in order to determine the output rate. Thus, for robots operating at high actuation frequencies an integrated operational amplifier, such as INA125P, coupled with a standard data acquisition (DAQ) box is recommended. In its current form, the entire measurement device can be constructed for under $£ 100$. At present the entire system is interfaced using an Arduino micro-controller which communicates with a PC via the serial port and a Python front-end program. This set up has been chosen specifically to make the system straightforward to use by students. A series of minimum working examples showing the inner workings of the hardware-software interface has been made available on Github, see [14].

\section{RIG CALIBRATION}

Prior to the employment of this set up for actual prototype testing, a calibration procedure had to be devised in order to achieve an estimate of the measurement accuracy both during steady and dynamic experiments.

Standard calibration protocols for dynamometers can be inexpensively performed by using a series of weights which exert known, constant loads on the force block and comparing the measured value to the applied one. However, in order to measure the dynamic thrust produced by a pulsed-jetting or flapping-foil vehicle, a purposely-designed procedure was required. This is meant to ensure that the system remains
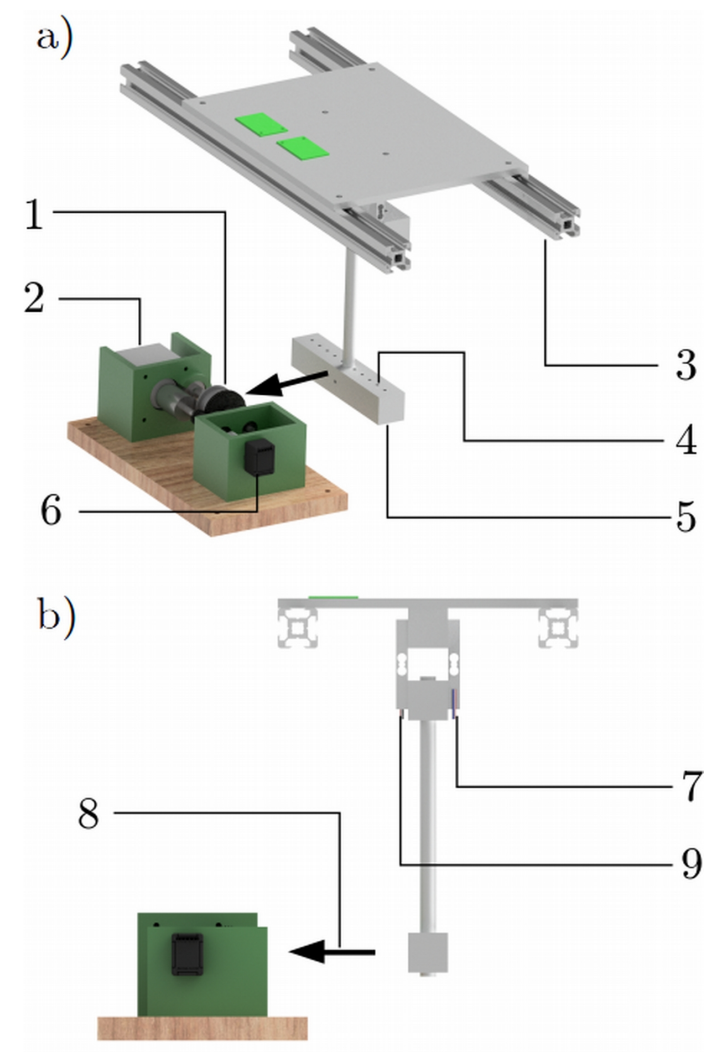

Fig. 3. Calibration set up showing (a) perspective view and (b) side view of the measurement rig and the calibration apparatus. Items refer to: (1) pulley, (2) stepper motor , (3) tank support for the measurement platform, (4) spring attachment points for moment calibration, (5) block for spring attachment points, (6) encoder, (7) transducer 1, (8) direction of tension load, (9) transducer 2. 


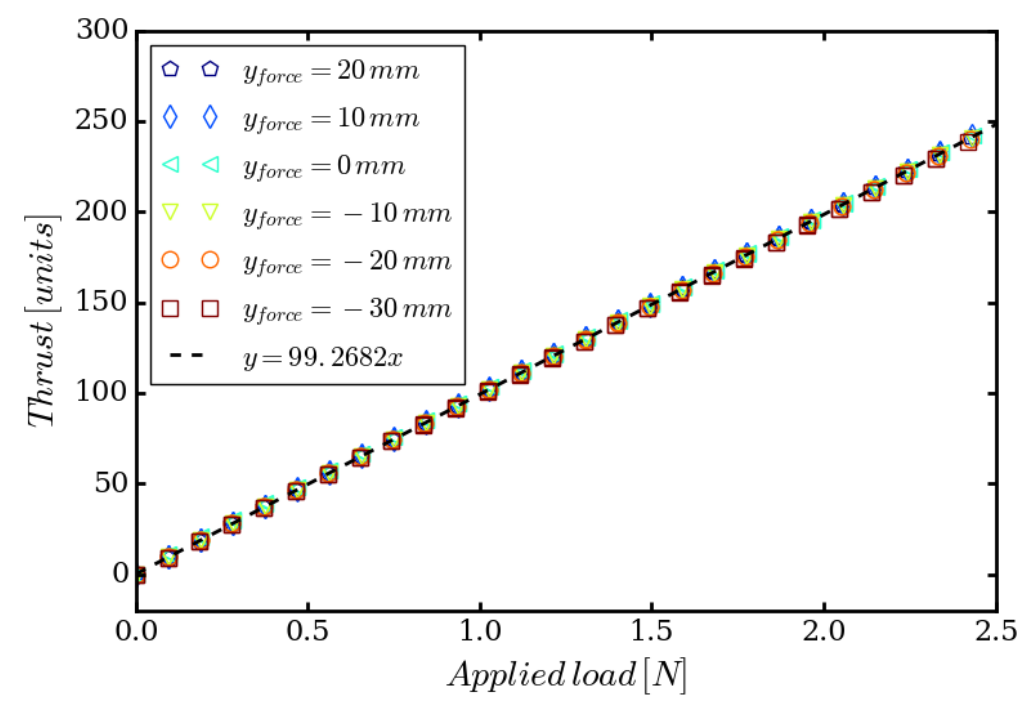

Fig. 4. Output from the steady linear loading (thrust) tests. Markers indicated recorded values and the dashed line is a linear regression fit used to derive the calibration constant. The y-axis represents the dimensionless output from the analog-to-digital converter and the units of the correlation constant is $[1 / \mathrm{N}]$. Different $y_{\text {force }}$ values denote the separation between the measurement axis and the point of application of the known force vector.

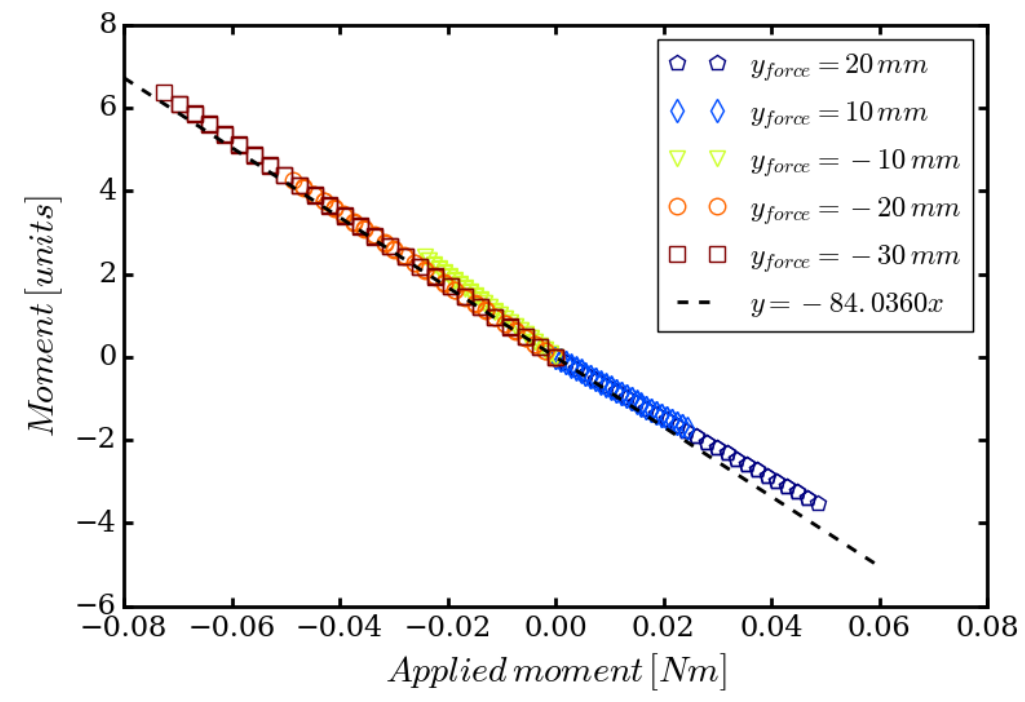

Fig. 5. Output from the steady moment tests. Markers indicated recorded values and the dashed line is a linear regression fit used to derive the calibration constant. The $\mathrm{y}$-axis represents the dimensionless output from the analog-to-digital converter and the units of the correlation constant is [1/Nm].

accurate when rapid impulses are imposed upon it. To do so, a calibration apparatus shown in Fig. 2 is employed. This is capable of generating unsteady loads of known magnitude at a controllable rate comparable to that of the pulsed-jetting routine of the robot.

This purpose-designed apparatus is manufactured and arranged in combination with the measurement rig depicted in Fig. 3. This consists of a platform comprising of a stepper motor, an encoder, a pulley, and a perforated aluminium block, item (5) in Fig. 3, fitted to the end of the cylindrical supporting rod. The pulley is connected via a spring of known elastic constant to the perforated block and hooked to one of the five attachment points present on either side of the cylindrical rod that will ultimately support the robot. Actuation of the stepper motor drives the rotation of the pulley which, in turn, stretches the spring. By attaching the spring directly on to the cylindrical rod a linear load is generated. This may be offset from the axis of the measurement system in order to apply a known torque. Using the known spring constant, the tension applied can be computed via the extension of the spring and hence a constant extensionper-motor-step can be derived. This allows to relate motor step rotation to the load exerted, both in steady and unsteady conditions.

\section{A. Steady force measurement}

The first step of calibration was to find an estimate for the calibration constants of the load cells. These scaled the force readings given by the transducers. These were found by applying a series of known loads by displacing the pulley from the zero position to its maximum angle while taking readings at fixed time intervals. For each position, a force 


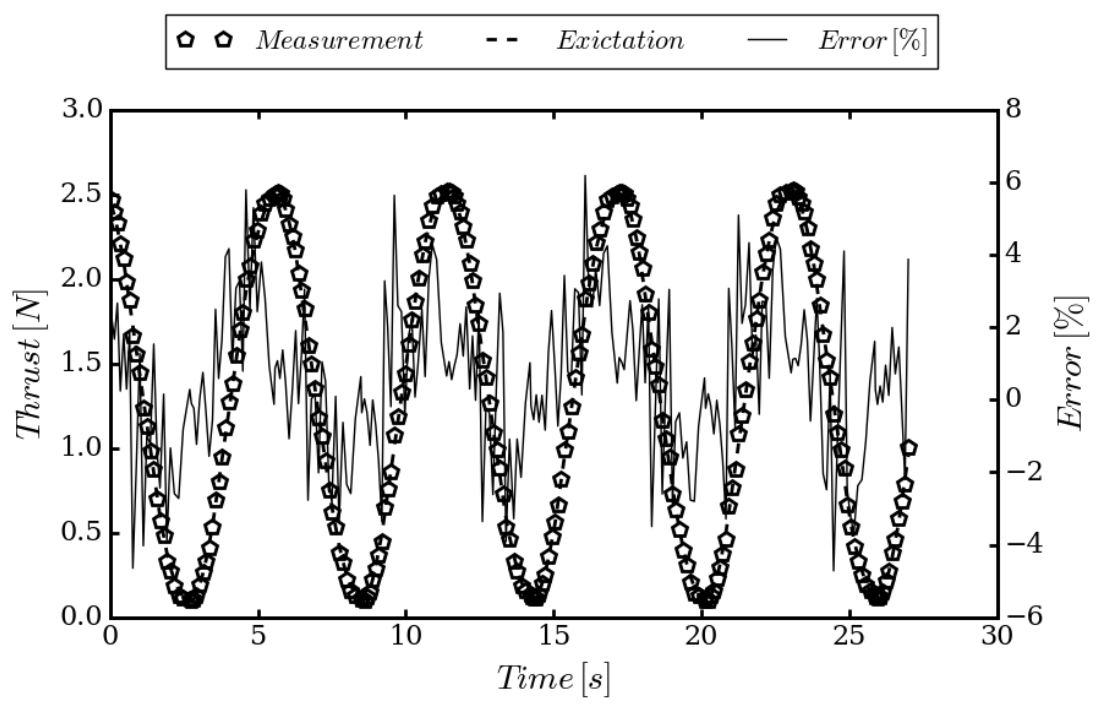

Fig. 6. Output from the dynamic linear loading tests with harmonic input force: markers represent the measurements from the load cells, the dashed line represents the load exerted by the stepper motor and the continuous line represents the error between the recorded and actual signal relative to the time-averaged load.

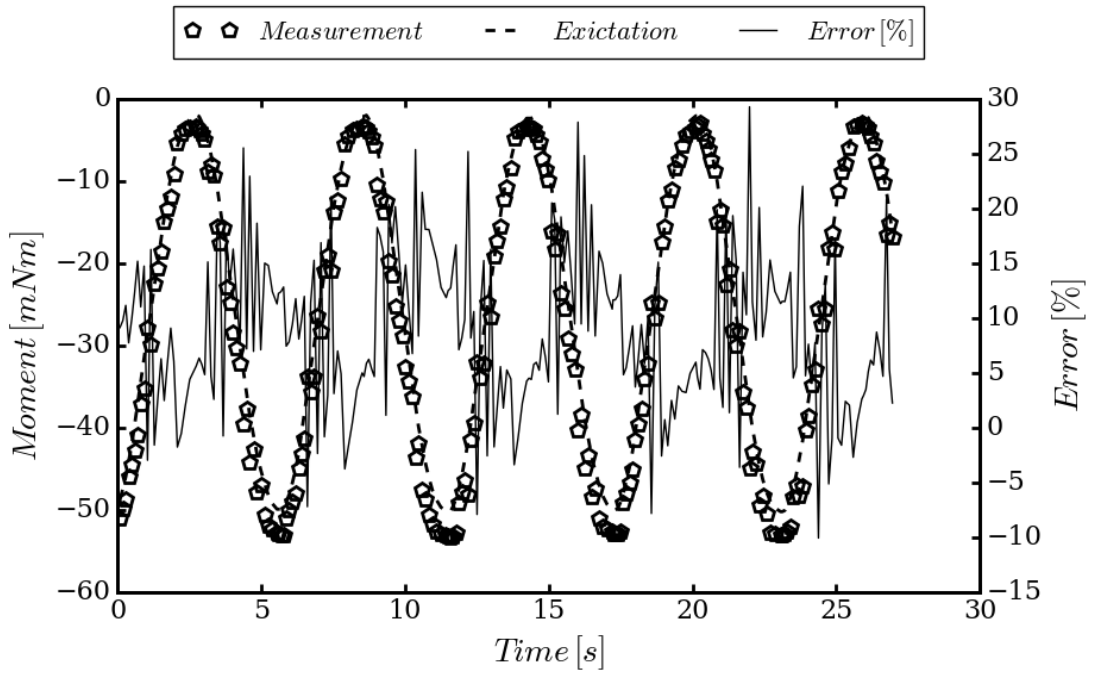

Fig. 7. Output from the dynamic moment tests with harmonic input force: markers represent the measurements from the load cells, the dashed line represents the load exerted by the stepper motor and the continuous line represents the error between the recorded and actual signal relative to the time-averaged load.

reading was found, and an error was calculated from this and the estimated load calculated from the extension of the spring. The process was then repeated by taking measurements as the pulley was returned to its neutral position. The calibration constant could then be found by fitting a linear regression curve to the collected data, as seen in Fig. 4.

In order to calibrate the yaw moment measurement, the spring was attached to various transverse positions and the same loading and unloading procedure was followed. The result may be seen in Fig. 5. The recorded values still follow a linear trend, although there is a degree of deviation, particularly as negative attachment positions of the spring are used. This was caused by a pure moment not having been applied, rather a force and a moment were employed at the same time. This effect was further amplified by possible inaccurate alignment of the force vector relative to the axis of the dynamometer or imperfect symmetry of the rig. In the range of values relevant to the current application between $\pm 0.01 \mathrm{Nm}$, however, the deviation lies below the $5 \%$ threshold and was assessed in more detail during the unsteady calibration.

\section{B. Unsteady force measurement}

Calibration for the time-varying experimental test was done by displacing the pulley in such a manner as to induce sinusoidal displacement of the end of the spring, which resulted in a sinusoidal excitation of the force block. The frequency of the applied sine wave was increased in order to check whether the load cells could be accurate for very sharp impulses of force. The range of actuation speed upon which calibration was performed was limited by the maximum operational speed of the stepper motor and gear system used, 


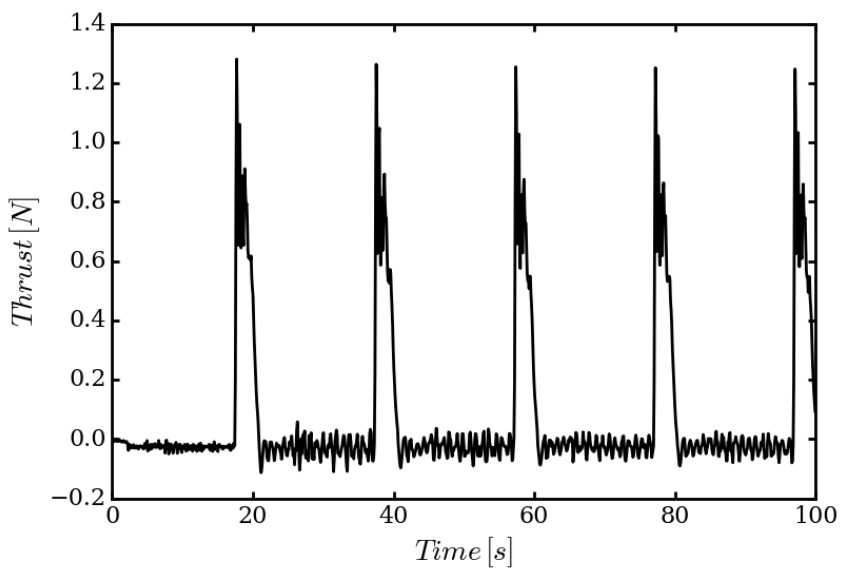

Fig. 8. Thrust output from a sequence of five pulsed-jets performed by the prototype of Fig. 10.

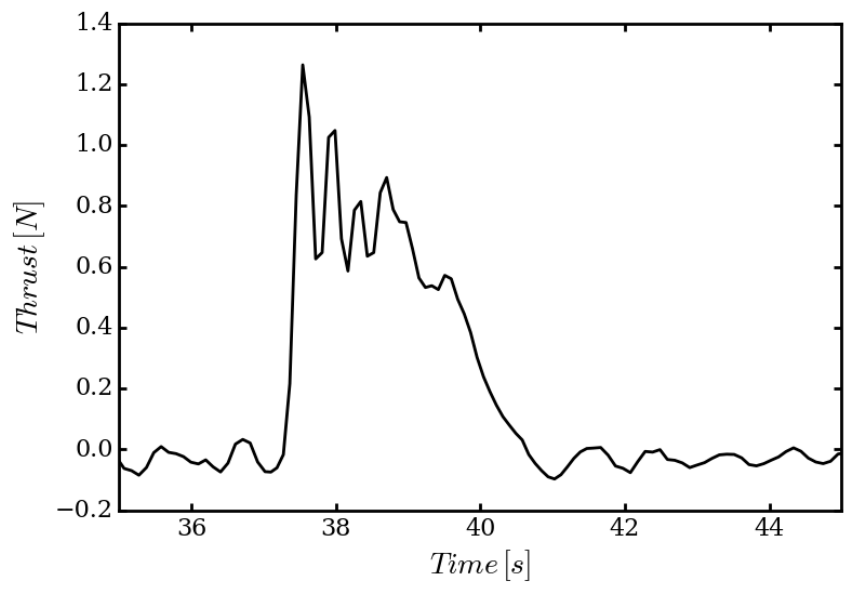

Fig. 9. Detail of the thrust output from a single pulsed-jet performed by the prototype of Fig. 10.

but revealed that waves with periods as low as $2 \mathrm{~s}$ could be captured without any noticeable increase in errors. The tests were also performed for each spring position to allow the error of unsteady moment measurements to be analysed as well.

The results are shown in Figs. 6 and 7 for a sinusoidal excitation with the period of $6 \mathrm{~s}$ with the spring attached $20 \mathrm{~mm}$ off the centreline of the rig. The relative error of the measured thrust varied between $\pm 4.5 \%$, while for the moment it peaked at $25 \%$ and has a mean absolute value of 9.4\%. These figures were computed using the mean value of the signal over the recorded five cycles. With a mean error of $\pm 1.7 \%$, the measurements of the unsteady thrust are sufficiently accurate to be employed in applications with low magnitudes of the applied forces, as it is the case with the thrust output the rig is intended for. The discrepancy in the moment reading, with a mean error of $10.2 \%$ and $9.4 \%$ respectively for the steady and dynamic cases, is greater than what would be considered acceptable for quantitative timeseries analysis. One of the future developments of the system is a further investigation of the exact source of these errors. At present, however, the thrust measurements are of interest and hence the moment reading is mostly used to determine the alignment of the robot in the horizontal plane, for which the present system is deemed suitable.

\section{ROBOT DESIGN}

The robot consists of a soft-bodied vehicle resemblant of a cephalopod (i.e. a squid or an octopus), similar to those presented in [15] and [16]. The vehicle working principle entails a cyclic sequence of inflation and deflation which drives a periodic pulsed-jetting routine. This kind of routine is aimed at benefiting from both the thrust generated by the expelled jet as well as the added-mass-variation force associated with the shape variation [17], [18]. During inflation, ambient fluid is pumped into the elastic body of the robot, charging the flexible walls with elastic potential energy. Throughout this stage, outflow of the internal fluid is prevented at the nozzle exit by a controllable valve. Once the elastic body has been inflated, the valve is opened, allowing the elastic walls to shrink while pushing the fluid across the nozzle and generating net forward momentum.

The prototype comprises of a $250 \mathrm{~mm}$ hollow elastic membrane of ellipsoidal shape truncated at $75 \%$ of its length and $4 \mathrm{~mm}$ thick. At the truncation location, the membrane is fitted onto a 3D printed funnel which acts as the stern of the vehicle and hosts the outflow valve and the pump, as seen in Fig. 1. A centrifugal M510 series TCS-micropump, item (7) in Fig. 1, capable of delivering $8.7 \mathrm{~L} / \mathrm{min}$ at $586 \mathrm{mbar}$ is employed to inflate the elastic shell walls. In order to
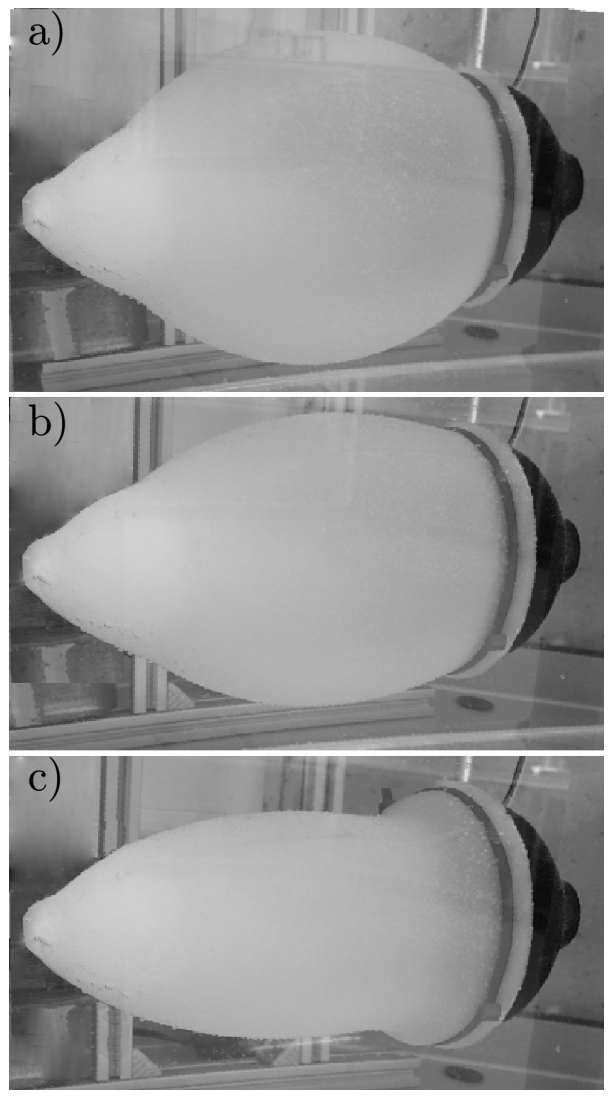

Fig. 10. The prototype during the pulsation routine displayed in Fig. 9 at (a) $37.0 \mathrm{~s}$, (b) $39.0 \mathrm{~s}$ and (c) $41.0 \mathrm{~s}$. 


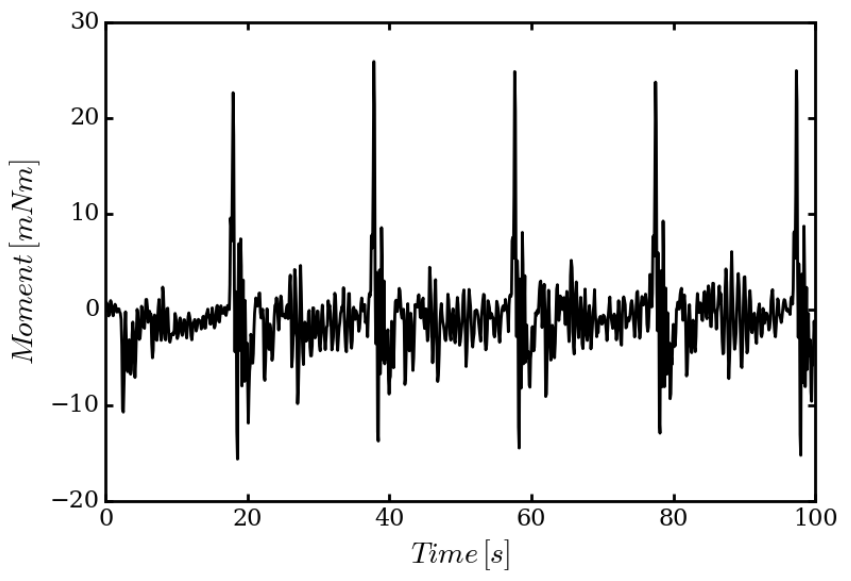

Fig. 11. Moment output from the same sequence of pulsed-jeting of Fig. 8.

regulate the outflow across the nozzle, a purpose 3D printed ball-valve is actuated by a Traxxas 2080 waterproof servo, item (8) in Fig. 1, via a spur gear, item (10) in Fig. 1.

\section{ROBOT TESTING}

Actuation of the vehicle is based on the repetition of the following routine: closing of the ball valve, activation of the pump, inflation of the elastic membrane, deactivation of the pump, opening of the ball valve. Thrust measurement associated with a sequence of pulsed-jets obtained from this actuation routine is reported in Fig. 8, with details of a single pulsation shown in Fig. 9. The shape variation which the vehicle undergoes during a single pulsed-jet such as that of Fig. 9 is presented in Fig. 10.

The data show repeatable cyclic bursts of thrust caused by the expulsion of water out of the back of the vehicle. Each of the actuations may be seen to lead to a sharp rise of the thrust as the valve is opened, followed by an approximately 3 second period of constant thrust. The forward force then decreases steadily to zero as the potential energy of the flexible walls of the robot is depleted. It may also be seen that the net yaw moment developed by the vehicle is approximately zero, suggesting it is aligned with the thrust measurement axis of the measurement rig. The recorded signal does show a positive bias when the valve is first opened, however. This suggests that the initial jet of liquid expelled from the vehicle is deflected by the rotating valve, leading to a small turning moment being developed. Finally, the ever-present fluctuations of the recorded signals suggest that the actuation of the robot induces circulation in the small water tank the tests were carried out in. Future tests are therefore scheduled to take place in a larger facility where boundary effects should be minimised.

\section{CONCLUSIONS}

We have introduced an apparatus intended for unsteady thrust characterization of a soft unmanned underwater vehicle which can readily be employed for more general propulsion systems. The employment of this experimental rig follows a protocol of calibration for the linear force and moments which guarantees an optimal error constrain both in steady and dynamic test-cases. The key strength of this experimental rig in the frame of soft robots prototyping lies in its ease of operation, the low cost of manufacturing and the chance to accommodate for additional DOFs as well as to implement it in self-propulsive platforms.

We have used this new experimental platform to measure the unsteady thrust production of a novel pulsed-jet softbodied aquatic vehicle. This has provided for the first time an accurate, time-dependent characterization of the complex propulsion routine of such unconventional soft robots.

\section{REFERENCES}

[1] B. Trimmer, "Soft robots," Current Biology, vol. 23, pp. 639-641, 2013.

[2] S. Saimek and P. Y. Li, "Motion planning and control of a swimming machine," International Journal of Robotics Research, vol. 23, pp. 27-53, 2004.

[3] J. Conte, Y. Modarres-Sadeghi, M. Watts., F. Hover, and M. Triantafyllou, "A fast-starting robotic fish that accelerates at $40 \mathrm{~ms}^{-2}$," Bioinspiration \& Biomimetics, vol. 5, 2010.

[4] H. Godaba, J. Li, Y. Wang, and J. Zhu, "A soft jellyfish robot driven by a dielectric elastomer actuator," IEEE Robotics and Automation Letters, vol. 1, no. 2, pp. 624-631, July 2016.

[5] F. Giorgio-Serchi, A. Arienti, F. Corucci, M. Giorelli, and C. Laschi, "Hybrid parameter identification of a multi-modal undwater soft robot," Bioinspiration \& Biomimetics, vol. 12, no. 2, p. 025007, 2017.

[6] M. Calisti, G. Picardi, and C. Laschi, "Fundamentals of soft robot locomotion," Journal of The Royal Society Interface, vol. 14, no. 130, 2017. [Online]. Available: http://rsif.royalsocietypublishing.org/ content/14/130/20170101

[7] J. A. Abbott, K. E. Peyer, M. C. Lagomarsino, L. Zhang, L. Dong, I. K. Kaliakatsos, and B. J. Nelson, "How should microrobots swim?" The International Journal of Robotics Research, vol. 28, pp. 1434-1447, 2009.

[8] J. Yu, R. Ding, Q. Yang, M. Tan, W. Wang, and J. Zhang, "On a bio-inspired amphibious robot capable of multimodal motion," IEEE Transactions on Mechatronics, vol. PP, pp. 1-10, 2011.

[9] M. Sfakiotakis, A. Kazakidi, A. Chatzidaki, T. Evdaimon, and D. P. Tsakiris, "Multi-arm robotic swimming with octopus-inspired compliant web," in IEEE/RSJ International Conference on Intelligent Robots and Systems, Chicago, IL, Sept 2014.

[10] M. Giorelli, F. Giorgio-Serchi, and C. Laschi, "Forward speed control of a pulsed-jet soft-bodied underwater vehicle," in MTS-IEEE OCEANS, San Diego, CA, USA, September 2013.

[11] F. Giorgio-Serchi, F. Renda, M. Calisti, and C. Laschi, "Thrust depletion at high pulsation frequencies in underactuated, soft-bodied, pulsed-jet vehicles," in MTS/IEEE OCEANS, Genova, Italy, May 2015.

[12] F. Renda, F. Giorgio-Serchi, F. Boyer, and L. C., "Modelling cephalopod-inspired pulsed-jet locomotion for underwater soft robots," Bioinspiration \& Biomimetics, vol. 10, 2015.

[13] F. Giorgio-Serchi and G. D. Weymouth, "Underwater soft robotics, the benefit of body-shape variations in aquatic propulsion," in Soft Robotics: Trends, Applications and Challanges, ser. Biosystems \& Biorobotics. Springer, 2016, vol. 17, pp. 37-46.

[14] A. Lidtke, "Arduino interfaces: a set of libraries in c++ and python to allow arduino to easily talk to sensors, actuators and a laptop," https://github.com/UnnamedMoose/ArduinoInterfaces, 2017.

[15] G. Weymouth, V. Subramaniam, and M. Triantafyllou, "Ultra-fast escape maneuver of an octopus-inspired robot," Bioinspiration \& Biomimetics, vol. 10, pp. 1-7, 2015.

[16] F. Giorgio-Serchi, A. Arienti, and C. Laschi, "Underwater soft-bodied pulsed-jet thrusters: Actuator modeling and performance profiling," International Journal of Robotics Research, 2016.

[17] G. Weymouth and M. Triantafyllou, "Ultra-fast escape of a deformable jet-propelled body," Journal of Fluid Mechanics, vol. 721, pp. 367385, 2013.

[18] F. Giorgio-Serchi and G. D. Weymouth, "Drag cancellation by addedmass pumping," Journal of Fluid Mechanics, vol. 798, 2016. 